\title{
THE RISE OF SN 2014J IN THE NEARBY GALAXY M82
}

\author{
A. Goobar ${ }^{1}$, J. Johansson ${ }^{1}$, R. Amanullah ${ }^{1}$, Y. CaO ${ }^{2}$, D. A. Perley ${ }^{2,21}$, M. M. Kasliwal ${ }^{3}$, R. Ferretti ${ }^{1}$, P. E. Nugent ${ }^{4,5}$, \\ C. Harris ${ }^{4,5}$, A. Gal-Yam ${ }^{6}$, E. O. OfeK ${ }^{6}$, S. P. TendulKar ${ }^{2}$, M. Dennefeld $^{7}$, S. VAlenti $^{8,9}$, I. ArCAVi $^{8,10}$, \\ D. P. K. Banerjee ${ }^{11}$, V. Venkataraman ${ }^{11}$, V. Joshi ${ }^{11}$, N. M. AshoK ${ }^{11}$, S. B. Cenko ${ }^{12,13}$, R. F. Diaz ${ }^{14}$, C. Fremling ${ }^{15}$, \\ A. Horesh ${ }^{6}$, D. A. Howell ${ }^{8,9}$, S. R. Kulkarni ${ }^{2}$, S. Papadogiannakis ${ }^{1}$, T. Petrushevska ${ }^{1}$, D. Sand ${ }^{16}$, \\ J. Sollerman ${ }^{15}$, V. Stanishev ${ }^{17}$, J. S. Bloom ${ }^{5}$, J. Surace ${ }^{18}$, T. J. Dupur ${ }^{19}$, and M. C. Liu ${ }^{20}$ \\ ${ }^{1}$ The Oskar Klein Centre, Physics Department, Stockholm University, Albanova University Center, SE 10691 Stockholm, Sweden; ariel@ @ysik.su.se \\ ${ }^{2}$ Cahill Center for Astrophysics, California Institute of Technology, Pasadena, CA 91125, USA \\ ${ }^{3}$ Observatories of the Carnegie Institution for Science, 813 Santa Barbara Street, Pasadena, CA 91101, USA \\ ${ }^{4}$ Computational Cosmology Center, Computational Research Division, Lawrence Berkeley \\ National Laboratory, 1 Cyclotron Road, MS 50B-4206, Berkeley, CA 94720, USA \\ ${ }^{5}$ Department of Astronomy, University of California Berkeley, B-20 Hearst Field Annex \# 3411, Berkeley, CA 94720-3411, USA \\ ${ }^{6}$ Department of Particle Physics and Astrophysics, Weizmann Institute of Science, Rehovot 76100, Israel \\ ${ }^{7}$ CNRS, Institut d'Astrophysique de Paris (IAP) and University P. et M. Curie (Paris 6), 98bis Boulevard Arago, F-75014 Paris, France \\ ${ }^{8}$ Las Cumbres Observatory Global Telescope Network, 6740 Corona Drive, Suite 102, Goleta, CA 93117, USA \\ ${ }^{9}$ Department of Physics, University of California, Santa Barbara, Broida Hall, Mail Code 9530, Santa Barbara, CA 93106-9530, USA \\ ${ }^{10}$ Kavli Institute for Theoretical Physics, University of California, Santa Barbara, CA 93106, USA \\ ${ }^{11}$ Physical Research Laboratory, Ahmedabad 380 009, India \\ ${ }^{12}$ Astrophysics Science Division, NASA Goddard Space Flight Center, Mail Code 661, Greenbelt, MD 20771, USA \\ ${ }^{13}$ Joint Space Science Institute, University of Maryland, College Park, MD 20742, USA \\ ${ }^{14}$ Observatory of Geneva, University of Geneva 51 Chemin des Maillettes, 1290 Sauverny, Switzerland \\ 15 The Oskar Klein Centre, Astronomy Department, Stockholm University, Albanova University Center, SE 10691 Stockholm, Sweden \\ ${ }^{16}$ Physics Department, Texas Tech University, Lubbock, TX 79409, USA \\ ${ }^{17}$ CENTRA-Centro Multidisciplinar de Astrofísica, IST, Avenida Rovisco Pais, 1049-001 Lisboa, Portugal \\ ${ }^{18}$ Infrared Processing and Analysis Center, California Institute of Technology, Pasadena, CA 91125, USA \\ ${ }^{19}$ Harvard-Smithsonian Center for Astrophysics, 60 Garden Street, Cambridge, MA 02138, USA \\ ${ }^{20}$ Institute for Astronomy, University of Hawaii, 2680 Woodlawn Drive, Honolulu, HI 96822, USA \\ Received 2014 February 7; accepted 2014 February 21; published 2014 March 7
}

\begin{abstract}
We report on the discovery of SN 2014J in the nearby galaxy M82. Given its proximity, it offers the best opportunity to date to study a thermonuclear supernova (SN) over a wide range of the electromagnetic spectrum. Optical, nearIR, and mid-IR observations on the rising light curve, orchestrated by the intermediate Palomar Transient Factory, show that SN 2014J is a spectroscopically normal Type Ia supernova (SN Ia), albeit exhibiting high-velocity features in its spectrum and heavily reddened by dust in the host galaxy. Our earliest detections start just hours after the fitted time of explosion. We use high-resolution optical spectroscopy to analyze the dense intervening material and do not detect any evolution in the resolved absorption features during the light curve rise. Similar to other highly reddened SNe Ia, a low value of total-to-selective extinction, $R_{V} \lesssim 2$, provides the best match to our observations. We also study pre-explosion optical and near-IR images from Hubble Space Telescope with special emphasis on the sources nearest to the SN location.
\end{abstract}

Key words: dust, extinction - galaxies: individual (Messier 82) - supernovae: individual (SN 2014J)

\section{INTRODUCTION}

Type Ia supernovae (SNe Ia) are among the most luminous transient events at optical wavelengths and extremely valuable tools to measure cosmological distances; see Goobar \& Leibundgut (2011) for a recent review. Yet, SNe Ia close enough to allow for detailed scrutiny of their physical properties are very rare, especially in a galaxy like M82, the host of several recent core-collapse SNe (Mattila et al. 2013; Gendre et al. 2013). At an estimated distance to M82 of 3.5 Mpc (Dalcanton et al. 2009), SN 2014J is the closest identified SN Ia in several decades, possibly rivaled by SN 1972E in NGC 5253 (Ardeberg $\&$ de Groot 1973) and SN 1986G in NGC 5128 (Phillips et al. 1987). Thus, SN 2014J is exceptionally well-suited for followup observations in a wide range of wavelengths, from radio to gamma-rays. These have the potential to yield transformational new clues into the progenitor systems of SNe Ia, as well as the

\footnotetext{
${ }^{21}$ Hubble Fellow.
}

detailed properties of dust along the line of sight, key astrophysical unknowns for the study of the accelerated expansion of the universe.

There is strong evidence that $\mathrm{SNe}$ Ia arise from thermonuclear explosions of carbon-oxygen white dwarfs (WDs) in binary systems (Nugent et al. 2011; Bloom et al. 2012). However, the nature of the second star remains unclear. For a long time, the preferred scenario was the single degenerate (SD) model (Whelan \& Iben 1973), where a WD accretes mass from a hydrogen or helium rich donor star, thus becoming unstable while approaching the Chandrasekhar mass. The double-degenerate (DD) model involving the merger of two WDs (Tutukov \& Yungelson 1981; Iben \& Tutukov 1984; Webbink 1984) has gained considerable observational support in recent years; see, e.g., Wang \& Han (2012).

In this work, we search for potential signatures of an SD progenitor system, such as variable $\mathrm{Na} \mathrm{D}$ lines, precursor nova eruptions, features in the early light curve, radio emission, or a coincident source in pre-explosion in Hubble Space Telescope (HST) images. 

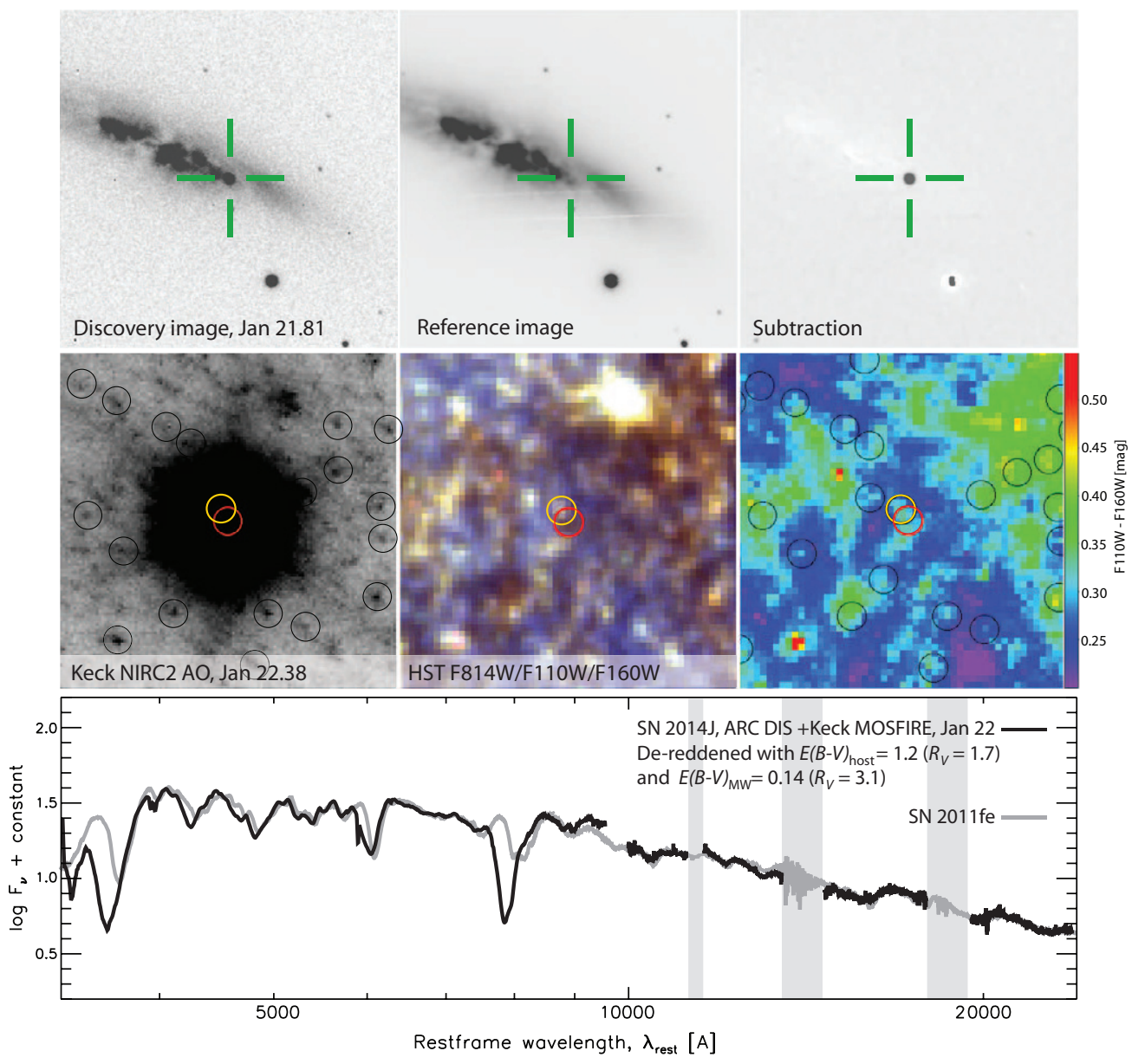

Figure 1. Top panels: $5^{\prime} \times 5^{\prime}$ sections of the discovery (Fossey et al. 2014), reference, and the subtraction images. Middle panels (from left to right): $5^{\prime \prime} \times 5^{\prime \prime}$ section of the Keck NIRC2 adaptive optics image used to match the SN coordinates (red circle) to the surrounding sources (black circles). The nearest resolved object (yellow circle) in the pre-explosion $H S T$ composite images $(F 815 \mathrm{~W} / F 110 \mathrm{~W} / F 160 \mathrm{~W})$ is offset by $0^{\prime \prime} 2$ from the best estimate of the SN position. The middle-right panel shows a color map $(F 110 \mathrm{~W}-F 160 \mathrm{~W})$ indicating the large scale structures, probably due to patches of dust. The bottom panel shows the first optical and NIR spectrum of SN 2014J and a comparison to a combined spectrum from SN 2011 fe by Pereira et al. (2013) and Hsiao et al. (2013), described in the text.

\section{DISCOVERY AND CLASSIFICATION}

SN 2014J was discovered by Fossey et al. (2014) in $B V$ $R$-band images of M82 obtained on 2014 January 21.81 UT at UCL's University of London Observatory. We have performed image subtractions using pre-explosion data from the Palomar P60 telescope as reference, calibrated with nearby stars listed in the APASS catalog 22 yielding a discovery magnitude of $R=10.99 \pm 0.03 \mathrm{mag}$. The discovery image (S. Fossey 2014, private communication) and the P60 reference image, as well as the difference between the two are shown in Figure 1, along with the pre-explosion HST images (GO:11360, PI: R. O'Conell; GO:10776, PI: M. Mountain). The relative position of SN 2014J with respect to neighboring stars (middle panel) was established using multiple short exposures in the $K$ band with adaptive optics and the NIRC2 wide camera at Keck (Tendulkar et al. 2014). In Section 5 we present a detailed analysis of the pre-explosion data.

A classification spectrum was obtained by the intermediate Palomar Transient Factory (iPTF) team on January 22.30 with the Dual Imaging Spectrograph on the ARC $3.5 \mathrm{~m}$ telescope (Cao et al. 2014), and in the near-IR using the MOSFIRE instrument at Keck. The combined spectra are shown in the bottom panel of Figure 1, while the photometry collected so far

\footnotetext{
22 http://www.aavso.org/apass
}

is displayed in Figure 2. The object shows characteristic spectral features associated with SNe Ia, e.g., similar to SN $2011 \mathrm{fe}$ (Pereira et al. 2013; Hsiao et al. 2013). However, the steep attenuation of the spectrum at short wavelengths is indicative of unusually large extinction by dust in the line of sight. A good match to the overall spectral energy distribution is found invoking a pronounced color excess, $E(B-V)_{\text {host }} \approx 1.2 \mathrm{mag}$, in addition to Galactic reddening, $E(B-V)_{\mathrm{Mw}}=0.14 \mathrm{mag}$ (Schlafly \& Finkbeiner 2011), as shown in Figure 3. For the comparison, the spectra of SN $2011 \mathrm{fe}$ were artificially reddened assuming a Milky Way type extinction law (Cardelli et al. 1989), where both the color excess and $R_{V}$ were allowed to vary freely. The spectrum favors a low value of the total-to-selective extinction, $R_{V} \lesssim 2$, as also suggested by spectropolarimetry observations by Patat et al. (2014). Low values of $R_{V}$ are not unusual in SNe Ia, especially in the cases of high extinction; see, e.g., Nobili \& Goobar (2008).

\section{THE iPTF-LED MULTI-WAVELENGTH MONITORING OF SN 2014J IN M82}

As a part of its continuous survey of the sky in the search for transients, iPTF has monitored M82 since 2009 October, with nearly daily cadence over the several months each year when M82 is visible from Palomar. The most recent campaign started on 2013 November 28. For the periods around full moon 


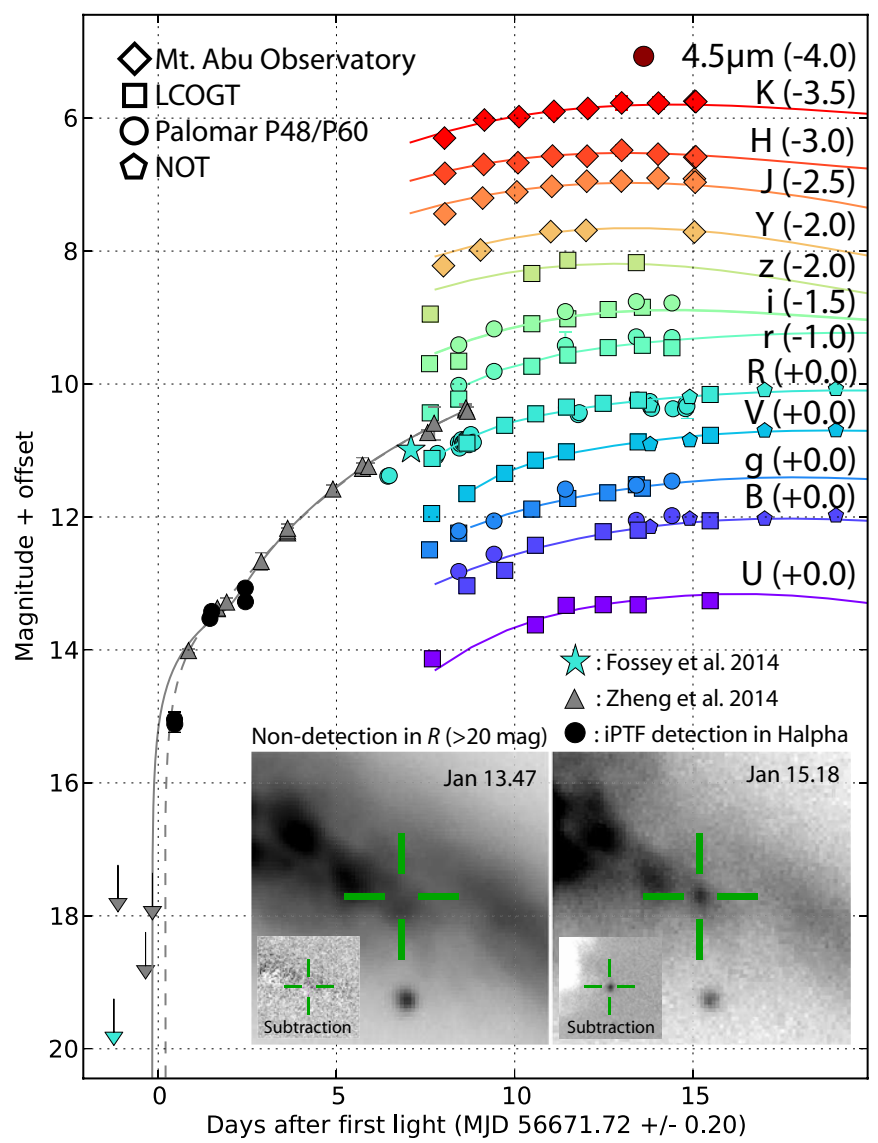

Figure 2. Light curves showing the rise of SN 2014J, along with SNooPy fits described in the text. The first P48 $H_{\alpha}^{656}$ and $H_{\alpha}^{663}$ narrowband detections are shown (black circles and inset image), S-corrected (Stritzinger et al. 2002) to the $R$ band. Due to lack of accurate absolute calibration for the $H_{\alpha}$ filters, a common offset was applied to connect with the data points in the pre-discovery light curve presented in Zheng et al. (2014). We also show their two fits of $t_{0}$, suggesting our first detection could be within $\sim 5 \mathrm{hr}$ from the onset of the supernova.

(e.g., around the time SN 2014J exploded), not well suited for transient searches, $H \alpha$ narrowband imaging was conducted.

The current best fit of the time of explosion, $t_{0}$, was reported by KAIT (Zheng et al. 2014) to be January 14.72 UT ( \pm 0.2 days). Upon later scrutiny of the pre-discovery P48 data, the SN was found in several observations from the iPTF $H \alpha$ narrowband survey, starting just hours after the fitted $t_{0}$. We find a relative flux increase from January 15.18 to January 16.18 of $1.6 \mathrm{mag}$, consistent with the "method $2 \mathrm{fit}$ " in Zheng et al. (2014). The $\mathrm{SN}$ is also prominent on $R$-band photometry from the P48 prior to January 21 shown in Figure 2, but remained undetected by our automated software due to pixel saturation.

Through an iPTF-led effort, involving also the Las Cumbres Observatory Global Telescope (LCOGT) network (Brown et al. 2013), the Nordic Optical Telescope, and the Mount Abu Observatory (Venkataraman et al. 2014), we have secured optical, near-IR, and mid-IR light curves carefully monitoring the rise of the SN, as shown in Figure 2. The $4.5 \mu$ m observations were taken under the Spitzer InfraRed Intensive Transients Survey (SPIRITS; PI: Kasliwal).

The spectra shown in Figure 3 are consistent with those from a normal Type Ia explosion, similar to, e.g., SN 2011fe, but reddened following a CCM law (Cardelli et al. 1989) with $E(B-V) \sim 1.2 \mathrm{mag}$ and $R_{V}=1.3-2$, in addition to Galactic reddening. Figure 2 also shows light curve fits using the SNooPy fitter (Burns et al. 2011) of the photometric data prior to maximum brightness. Best fits are found for $E(B-V)_{\text {host }}=1.22 \pm 0.05 \mathrm{mag}$ and $R_{V}=1.4 \pm 0.15$. We expect the accuracy of the fitted parameters to improve, as the light curve shape estimate will profit from the decreasing part of the SN light curve. However, the available data clearly puts $R_{V}$ well below the Galactic average value, $R_{V}=3.1$.

We also obtained two $1800 \mathrm{~s}$ high-resolution $(R=40,000)$ spectra with SOPHIE at Observatorie Haute-Provence on January 26.0 and January 28.0. Further, two $1800 \mathrm{~s}$ spectra $(R=67,000)$ were obtained with the FIbre-fed Echelle Spectrograph (FIES) on January 27.3 and another on February 1.0 with the Nordic Optical Telescope.

All spectra reveal deep multiple component $\mathrm{Na}$ I D absorption and diffuse interstellar bands (DIBs), including $\lambda \lambda 5780,5797,6284$, and 6614 , also reported by Cox et al. (2014) and Kotak (2014). The SOPHIE spectra further contain well resolved $\mathrm{Ca}$ II $\mathrm{H} \& \mathrm{~K}$ with features matching those of the $\mathrm{Na}$ I D lines, shown in the co-added spectrum in Figure 4. We have not detected any significant time evolution for any of the resolved components of the $\mathrm{Na}$ I $\mathrm{D}$ doublet over the four epochs (at the $\sim 10 \%$ level for $3 \sigma$ ), thus motivating the combination of the spectra.

Following the procedure outlined by Phillips et al. (2013), we measure the EW of the $\lambda 5780 \mathrm{DIB}$ to derive an independent estimate of host galaxy extinction for SN 2014J. We find $\mathrm{EW}(5780)=0.48 \pm 0.01 \AA$ corresponding to $A_{V}^{\text {host }}=2.5 \pm 1.3$ mag.

Given the low recession velocity of M82, it is difficult to separate the contribution from the Milky Way and the SN host galaxy absorption. However, the availability of $\mathrm{HI}$ data from the LAB survey ${ }^{23}$ in the direction of M82 (see inset panel in Figure 4; Kalberla et al. 2005) clearly indicates which features are Galactic. Hence, all the absorption features redshifted with respect to the Milky Way are due to intervening material in M82.

\section{SPECTRAL MODELING}

In Figure 3 we present a time-series spectral comparison between SN 2011fe and SN 2014J starting roughly 12 days before peak brightness. The SNe are remarkably similar in their spectral evolution. The main differences seen are that the overall velocities are higher in SN 2014J (see the inset Si II velocity plot) and there is a strong signature of high-velocity Si II and Ca II in this SN.

To further investigate these differences we carried out a set of SYNAPPS (Thomas et al. 2011) fits to these two SNe as well as to SN 2005cf, which was distinct in its pervasive high-velocity features (Wang et al. 2009). We present the results in Figure 5. In our fits to the red-side of the optical spectra we have employed the ions: C II, O I, Mg II, Si II, and Ca II with the latter two having both photospheric and high-velocity components. We see that SN 2014J more closely resembles SN 2005cf with respect to the high-velocity $\mathrm{Si}$ II and $\mathrm{Ca}$ II features which extend easily over the range of $20,000-30,000 \mathrm{~km} \mathrm{~s}^{-1}$. Unlike either SN $2005 \mathrm{cf}$ or SN 2011fe, C II and O I are absent at this phase in SN 2014J. We searched for the presence of C I $\lambda 1.0693 \mu \mathrm{m}$ line in our NIR spectrum, but the signal-to-noise is too low to do a meaningful fit comparable to that done for SN 2011fe (Hsiao et al. 2013). We do note that due to the stronger than average $\mathrm{Mg}$ II features seen in the optical for SN 2014J, this analysis may be more

\footnotetext{
23 http://www.astro.uni-bonn.de/en/download/data/lab-survey/
} 


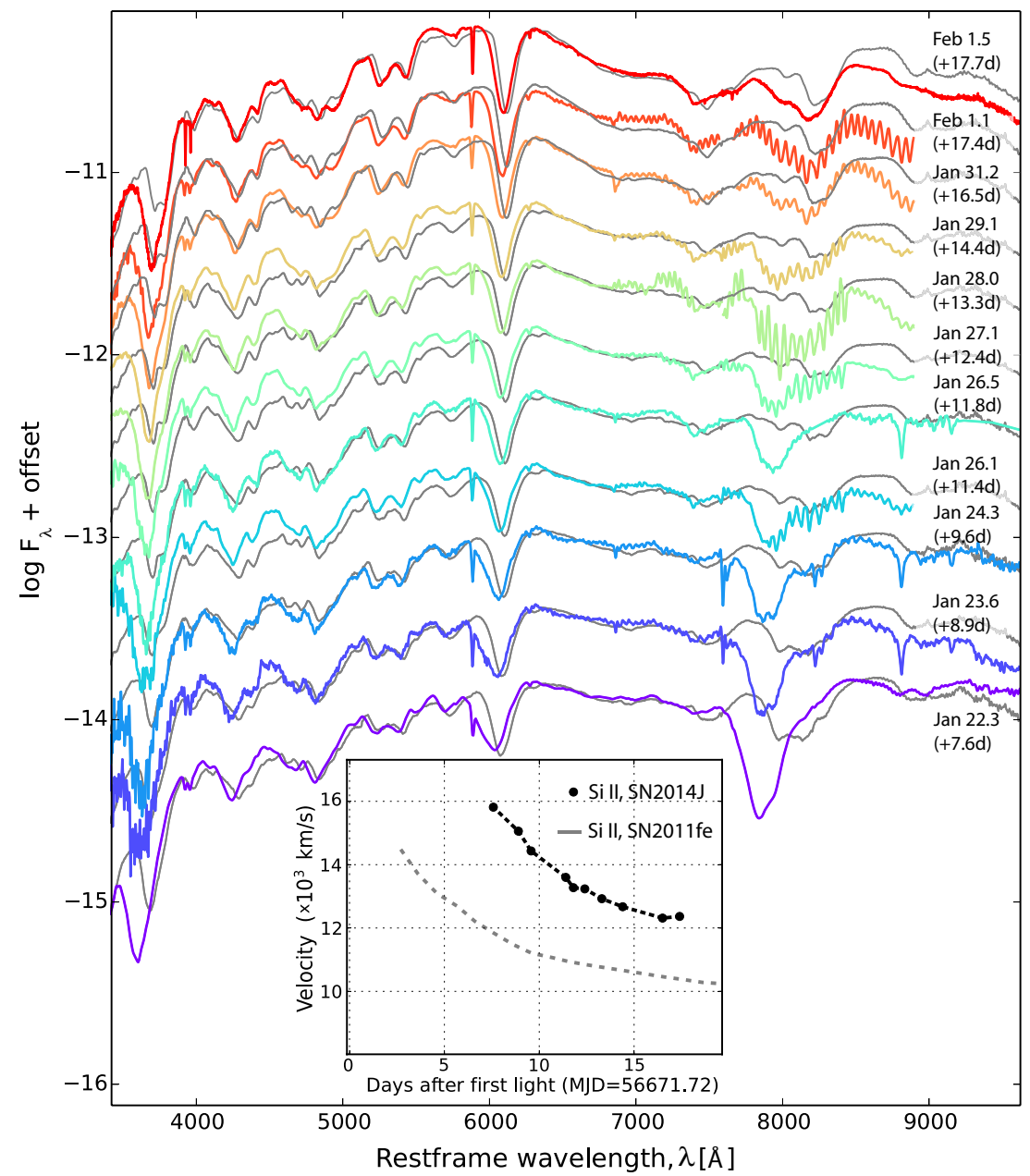

Figure 3. Pre-max spectroscopic follow-up of SN 2014J, starting 7.6 days from estimated supernova onset using the ARC 3.5 m DIS, LCOGT FLOYDS, NOT ALFOSC, and P200 DBSP spectrographs. Spectra of SN $2011 \mathrm{fe}$ (Pereira et al. 2013) at similar epochs (gray lines, reddened by $E(B-V)$ host $=1.2$ mag with $R_{V}=1.7$ and $E(B-V)_{\mathrm{MW}}=0.14$ mag with $R_{V}=3.1$ are shown for comparison). The inset panel shows that SN 2014J has higher Si II velocity than SN 2011fe and a steeper velocity gradient (Benetti et al. 2005).

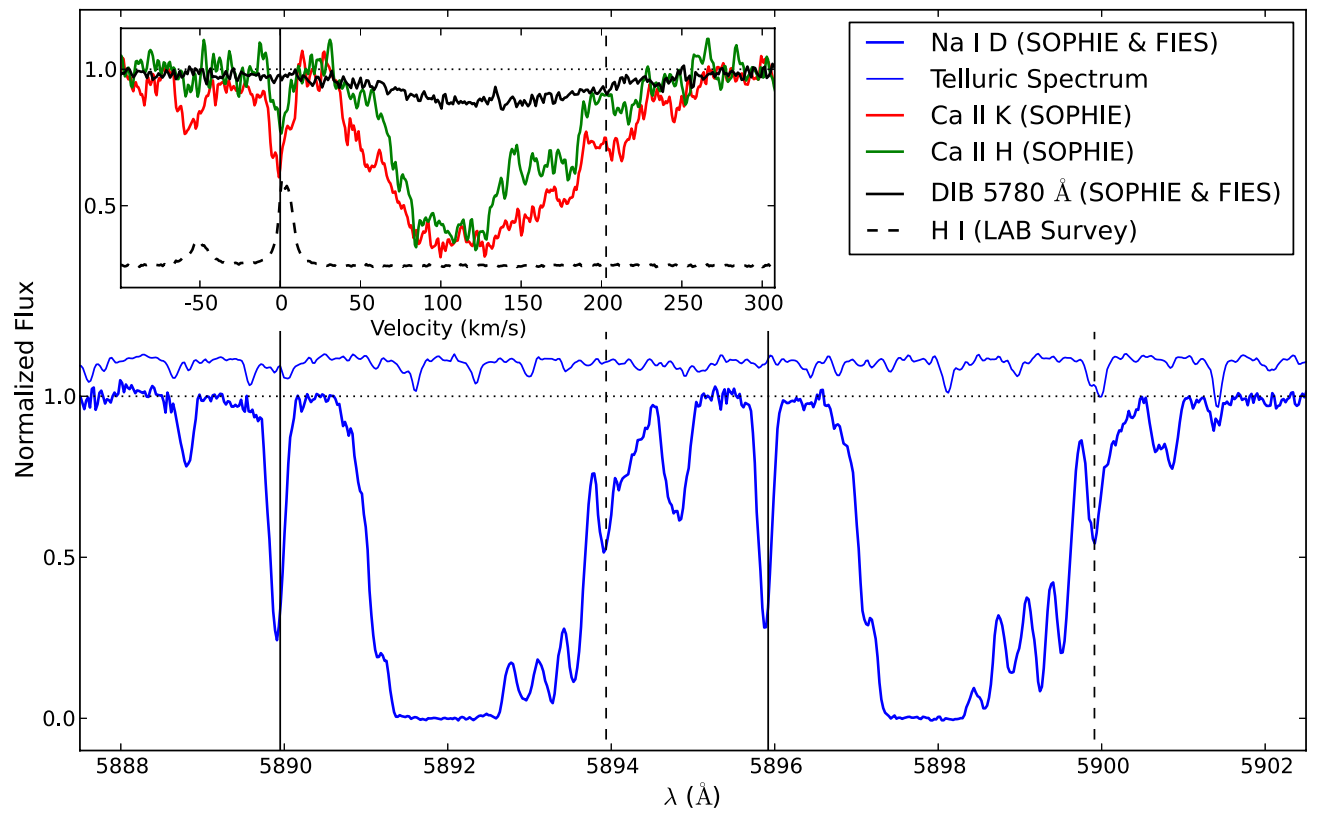

Figure 4. Normalized Na I D doublet, plotted with solid vertical lines indicating the Galactic rest-frame wavelengths and the dashed vertical lines correspond to the mean velocity of M82 $\left(203 \mathrm{~km} \mathrm{~s}^{-1}\right)$. The inset panel shows the velocity distribution of Ca II H \& K (green and red lines) and the $\lambda 5780$ DIB (black). Additionally, the $\mathrm{H}$ I $\lambda 21 \mathrm{~cm}$ emission spectrum of the line of sight of M82 from the LAB survey (Kalberla et al. 2005) is plotted (dashed gray line). The features of the Na I D and Ca II H \& K, at approximately -50 and $0 \mathrm{~km} \mathrm{~s}^{-1}$ with respect to the Galactic rest frame can be attributed to absorption in the Milky Way. The main Na I D absorption features, saturated between $74-135 \mathrm{~km} \mathrm{~s}^{-1}$, originate in M82. 


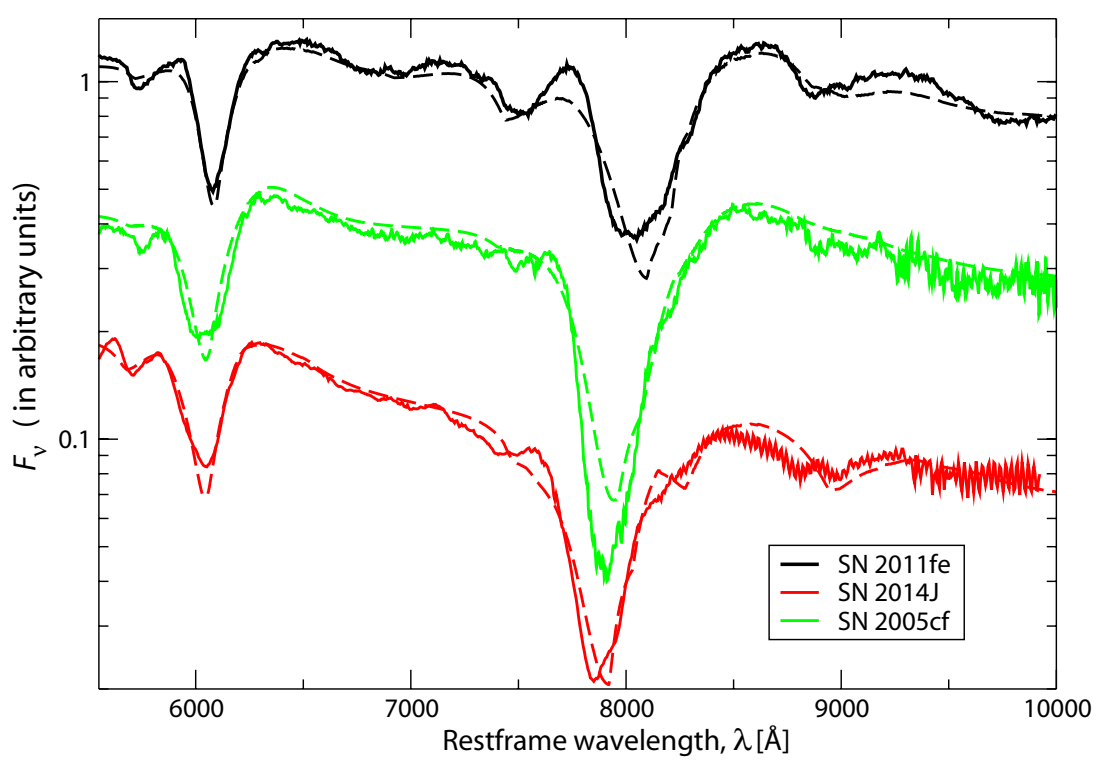

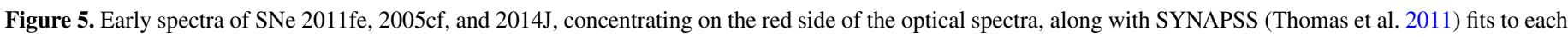

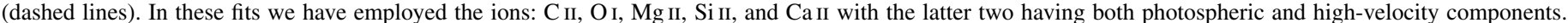

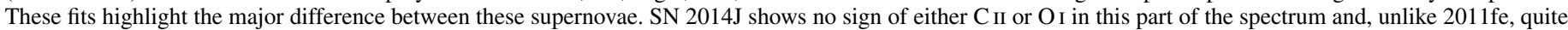

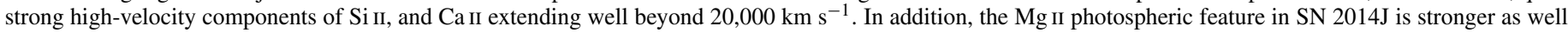

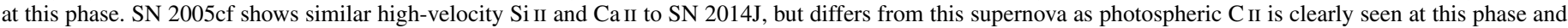

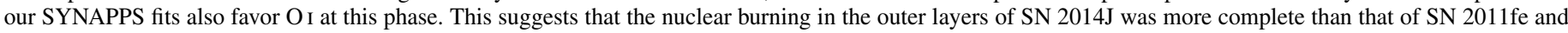
the lack of C II compared to SN 2005cf may imply the same or perhaps a viewing angle effect due to an off-center detonation (Parrent et al. 2011).

challenging, even with higher quality spectra, as the presence of Mg II $\lambda 1.0927 \mu \mathrm{m}$ should be quite strong as well.

Several explanations for the origin of the high-velocity features have been presented, from density enhancements via swept up circumstellar material (CSM) (Gerardy et al. 2004; Tanaka et al. 2006) to mixing or more complete burning in the outer layers of the SN (Mazzali et al. 2005a, 2005b) to ionization effects in the outer layers (Blondin et al. 2013). What is clear is that the features do offer a unique diagnostic for understanding properties of the progenitor system and/or the explosion mechanism and correlations between the strength of these features and the underlying colors and light curves of the SNe Ia (Childress et al. 2014; Maguire et al. 2013).

SN 2014J is among a class of SNe Ia where high-velocity features are present yet little to no evidence for $\mathrm{C}_{\text {II }}$ exists even in very early spectra (see the broad-line or high-velocity gradient examples in Parrent et al. 2011). Since extensive UV data from HST exists for both SNe 2005cf and 2011fe, it will be interesting to see which of these supernovae SN 2014J most closely matches with respect to both the color and luminosity evolution.

\section{THE QUEST FOR THE PROGENITOR SYSTEM}

M82 has been extensively imaged by HST, thus it is possible to study the environment of the SN prior to the explosion. Because of the large attenuation due to dust in the line of sight, we concentrate on the NIR bands. We perform aperture photometry on the nearest sources to SN 2014J, shown in Figure 1. The closest object (yellow circle in Figure 1) falls 0 '.2 from the current best estimate of the SN location (R.A. = $9^{\mathrm{h}} 55^{\mathrm{m}} 42^{\mathrm{s}} .217(1)$, decl. $=69^{\circ} 40^{\prime} 26^{\prime \prime} .56(4)$ in $\mathrm{J} 2000$ coordinates with respect to the HST image; Tendulkar et al. 2014), corresponding to a $4 \sigma$ spatial offset. For this source, we measure AB magnitudes of $F 110 \mathrm{~W}=21.4 \pm 0.4, F 128 \mathrm{~N}=21.9 \pm 0.4$, $F 160 W=20.6 \pm 0.4, F 164 N=21.2 \pm 0.4$. The error is dom- inated by the uncertain background subtraction as a result of source confusion. The $F 110-F 160 \mathrm{~W}$ color is typical of other sources near this position. At a distance of $3.5 \mathrm{Mpc}$, the corresponding absolute magnitude of the nearest resolved object is $J_{\mathrm{AB}} \sim H_{\mathrm{AB}} \sim-7 \mathrm{mag}\left(A_{H}<A_{J}<0.4 \mathrm{mag}\right)$. This source could represent a stellar cluster, a grouping of unrelated objects or a region of relatively low dust attenuation.

Next, we consider the possibility that the source is a donor star in the SD scenario. The derived luminosity would then suggest a very luminous red supergiant. However, in the case where an SN originates from a system with CSM created by a mass-loss from a donor star, the interaction between the SN ejecta and the CSM is expected to give rise to radio emission (Chevalier 1982, 1988). The radio null-detections on January 23 and 24 (Chomiuk et al. 2014; Chandler \& Marvil 2014) can therefore be used to derive an upper limit on the mass-loss rate. Adopting an SN shockwave velocity of $3 \times 10^{4} \mathrm{~km} \mathrm{~s}^{-1}$ (about twice the Si II velocity; see Figure 3), and similar parameters as assumed by Horesh et al. (2012) for SN 2011fe, the upper limit on the mass-loss rate is $\dot{M} \leqslant 7 \times 10^{-9}\left(w / 100 \mathrm{~km} \mathrm{~s}^{-1}\right) M_{\odot} \mathrm{yr}^{-1}$, where $w$ is the mass-loss wind velocity. The upper limit is comparable to the ones obtained for SN 2011fe (Horesh et al. 2012; Chomiuk et al. 2012). Given these tight limits and the spatial displacement, we conclude that the closest resolved source in the pre-explosion $H S T$ images is unlikely to be a donor star.

Finally, we note that the $F 110 W-F 160 W$ color map shown in the middle right panel of Figure 1 suggests that SN 2014J is at the edge of a dust patch, about $4 \mathrm{pc}$ in projected size. Light echoes may thus be expected for this $\mathrm{SN}$.

We have searched for possible nova outbursts in the historic P48 $R$-band data covering a period of about 1500 days prior to the detection of SN 2014J. By binning the data in bins of 15 days, we do not find any excess larger than $4 \sigma$ (calculated using the bootstrap technique; see Efron 1982). Our limiting magnitude is $R>19.5$ mag for a total time span of 1000 days, and $R>20.25$ mag for more than 765 days in this 1500 day time 
window. Assuming $A_{R}=2 \mathrm{mag}$, compatible with the extinction we estimate based on the $\mathrm{SN}$ colors, this corresponds to absolute magnitudes $M_{\mathrm{R}}=-10.2$ and -9.5 respectively at the distance of M82. However, given the uncertainties on the properties of recurrent novae, see, e.g., Tang et al. (2014), we refrain from drawing firm conclusions against the possibility of recurrent novae preceding SN 2014J based on these non-detections.

\section{SUMMARY AND CONCLUSIONS}

The discovery of SN 2014J presents us with a unique opportunity to explore the physics of Type Ia SNe and the lineof-sight effects due to intervening matter. Further understanding in these areas is of utmost importance for the use of SNe Ia in cosmology. The early data from P48, starting as early as only hours from the explosion, and the multi-wavelength follow-up by the iPTF team covers an important range of the available windows in the electromagnetic spectrum. Just as the light curve reaches its maximum, we have learned that the $\mathrm{SN}$ has suffered non-standard extinction. We have searched for, but not detected, any time variation in our high-resolution spectra of the $\mathrm{Na}$ I D doublet. Similarly, we do not detect any pre-explosion activity in the $\sim 1500$ days of P48 monitoring. In a study of pre-explosion $H S T$ images in the near-IR, the nearest resolved source is found 0 .'2 away from the $\mathrm{SN}$ location. The source brightness and offset from the SN makes it unlikely to be a donor star in a singledegenerate scenario.

Further, we make a first study of the spectral features of SN 2014J and find that it exhibits high-velocity features from intermediate mass material but lacks $\mathrm{C}$ and $\mathrm{O}$ often seen in very early spectra. Otherwise, it is a very similar to several wellstudied normal SNe Ia.

We thank $\mathrm{S}$. Fossey for making the discovery $R$-band image of SN 2014J available to us. We are grateful to S. Fossey and M. Phillips for helpful comments on the manuscript. We acknowledge A. McKay, A. Bradley, N. Scoville, P. L. Capak, C. M. Carollo, S. Lilly, H. Sheth, V. Bhalerao, P. Donati, S. Geier, F. Saturni, G. Nowak, and A. Finoguenov for cooperating with ToO observations. A.G. and R.A. acknowledge support from the Swedish Research Council and the Swedish Space Board and A.G.-Y. an ERC grant. M.M.K. acknowledges generous support from the Hubble Fellowship and Carnegie-Princeton Fellowship. Based on observations made with the Nordic Optical Telescope, operated by the Nordic Optical Telescope Scientific Association at the Observatorio del Roque de los Muchachos, La Palma, Spain, of the Instituto de Astrofisica de Canarias, Faulkes Telescope North image observed by Gain Lee, the Mount Abu $1.2 \mathrm{~m}$ Infrared telescope, India, and the $1.93 \mathrm{~m}$ telescope of Haute-Provence Observatory, CNRS, France. This research used resources of the National Energy Research Scientific Computing Center, which is supported by the Office of Science of the U.S. Department of Energy under Contract No. DE-AC02-05CH11231.

\section{REFERENCES}

Ardeberg, A., \& de Groot, M. 1973, A\&A, 28, 295

Benetti, S., Cappellaro, E., Mazzali, P. A., et al. 2005, ApJ, 623, 1011

Blondin, S., Dessart, L., Hillier, D. J., \& Khokhlov, A. M. 2013, MNRAS, 429, 2127

Bloom, J. S., Kasen, D., Shen, K. J., et al. 2012, ApJL, 744, L17

Brown, T. M., Baliber, N., Bianco, F. B., et al. 2013, PASP, 125, 1031

Burns, C. R., Stritzinger, M., Phillips, M. M., et al. 2011, AJ, 141, 19

Cao, Y., Kasliwal, M. M., \& McKay, A. 2014, ATel, 5786, 1

Cardelli, J. A., Clayton, G. C., \& Mathis, J. S. 1989, ApJ, 345, 245

Chandler, C. J., \& Marvil, J. 2014, ATel, 5812, 1

Chevalier, R. A. 1982, ApJL, 259, L85

Chevalier, R. A. 1988, Natur, 332, 514

Childress, M. J., Filippenko, A. V., Ganeshalingam, M., \& Schmidt, B. P. 2014, MNRAS, 437, 338

Chomiuk, L., Soderberg, A. M., Moe, M., et al. 2012, ApJ, 750, 164

Chomiuk, L., Zauderer, B. A., Margutti, R., \& Soderberg, A. 2014, ATel, 5800,1

Cox, N. L. J., Davis, B. A., Patat, F., \& Van Winckel, H. 2014, ATel, 5797, 1

Dalcanton, J. J., Williams, B. F., Seth, A. C., et al. 2009, ApJS, 183, 67

Efron, B. 1982, The Jackknife, the Bootstrap and Other Resampling Plans (Philadelphia, PA: Society for Industrial and Applied Mathematics (SIAM))

Fossey, S., Cooke, B., Pollack, G., Wilde, M., \& Wright, T. 2014, CBET, 3792, 1

Gendre, M. A., Fenech, D. M., Beswick, R. J., Muxlow, T. W. B., \& Argo, M. K. 2013, MNRAS, 431, 1107

Gerardy, C. L., Höflich, P., Fesen, R. A., et al. 2004, ApJ, 607, 391

Goobar, A., \& Leibundgut, B. 2011, ARNPS, 61, 251

Horesh, A., Kulkarni, S. R., Fox, D. B., et al. 2012, ApJ, 746, 21

Hsiao, E. Y., Marion, G. H., Phillips, M. M., et al. 2013, ApJ, 766, 72

Iben, I., Jr., \& Tutukov, A. V. 1984, ApJS, 54, 335

Kalberla, P. M. W., Burton, W. B., Hartmann, D., et al. 2005, A\&A, 440, 775

Kotak, R. 2014, ATel, 5816, 1

Maguire, K., Sullivan, M., Patat, F., et al. 2013, MNRAS, 436, 222

Mattila, S., Fraser, M., Smartt, S. J., et al. 2013, MNRAS, 431, 2050

Mazzali, P. A., Benetti, S., Altavilla, G., et al. 2005a, ApJL, 623, L37

Mazzali, P. A., Benetti, S., Stehle, M., et al. 2005b, MNRAS, 357, 200

Nobili, S., \& Goobar, A. 2008, A\&A, 487, 19

Nugent, P. E., Sullivan, M., Cenko, S. B., et al. 2011, Natur, 480, 344

Parrent, J. T., Thomas, R. C., Fesen, R. A., et al. 2011, ApJ, 732, 30

Patat, F., Taubenberger, S., Baade, D., et al. 2014, ATel, 5830, 1

Pereira, R., Thomas, R. C., Aldering, G., et al. 2013, A\&A, 554, A27

Phillips, M. M., Phillips, A. C., Heathcote, S. R., et al. 1987, PASP, 99, 592

Phillips, M. M., Simon, J. D., Morrell, N., et al. 2013, ApJ, 779, 38

Schlafly, E. F., \& Finkbeiner, D. P. 2011, ApJ, 737, 103

Stritzinger, M., Hamuy, M., Suntzeff, N. B., et al. 2002, AJ, 124, 2100

Tanaka, M., Mazzali, P. A., Maeda, K., \& Nomoto, K. 2006, ApJ, 645, 470

Tang, S., Bildsten, L., Wolf, W. M., et al. 2014, arXiv:1401.2426

Tendulkar, S. P., Liu, M. C., Dupuy, T. F., \& Cao, Y. 2014, ATel, 5789, 1

Thomas, R. C., Nugent, P. E., \& Meza, J. C. 2011, PASP, 123, 237

Tutukov, A. V., \& Yungelson, L. R. 1981, NInfo, 49, 3

Venkataraman, V., Banerjee, D. P. K., Joshi, V., Ashok, N., \& Bhalerao, V. 2014, ATel, 5793, 1

Wang, B., \& Han, Z. 2012, NewAR, 56, 122

Wang, X., Li, W., Filippenko, A. V., et al. 2009, ApJ, 697, 380

Webbink, R. F. 1984, ApJ, 277, 355

Whelan, J., \& Iben, I., Jr. 1973, ApJ, 186, 1007

Zheng, W., Shivvers, I., Filippenko, A. V., et al. 2014, arXiv:1401.7968 